\title{
INTERCALAÇÃO ELETROQUíMICA DE CÁTIONS HEXILAMÍNIO NA MATRIZ LAMELAR 1T-TiS
}

Fernando Wypych, Maria A. B. Gomes e Luciana B. Adad

Departamento de Química - Universidade Federal do Paraná - CP - 19081 - 81531-990 - Curitiba - Pr

Ireno Denicoló

Departamento de Física - Universidade Federal do Paraná - CP 19081 - 81531-990 - Curitiba - Pr

Recebido em 20/3/96; aceito em 8/8/96

\begin{abstract}
ELECTROCHEMICAL INTERCALATION OF HEXILAMINIUM CATIONS INTO THE LAYERED COMPOUND 1T-TiS ${ }_{2}$. The electrochemical synthesis of a ternary compound obtained by the intercalation of hydrated hexilaminium cations into the layered compound 1T-TiS ${ }_{2}$ is reported. Two different compounds were detected by cyclovoltammetry and studied by $\mathrm{X}$-ray diffractometry. Models showing the steric arrangement of the hydrated hexilaminium cations into the Van der Waals gaps were proposed.
\end{abstract}

Keywords: intercalation compounds; electrochemistry; titanium disulfide.

\section{INTRODUÇÃO}

Dissulfeto de titânio trigonal, $1 \mathrm{~T}-\mathrm{TiS}_{2}$, é um material de estrutura lamelar, no qual os átomos de titânio estão ligados covalentemente a seis átomos de enxofre formando um octaedro regular ${ }^{1}$. Estes octaedros são ligados uns aos outros pelas arestas formando lamelas bidimensionais as quais são ligadas umas às outras por forças fracas do tipo van der Waals (Fig. 1).

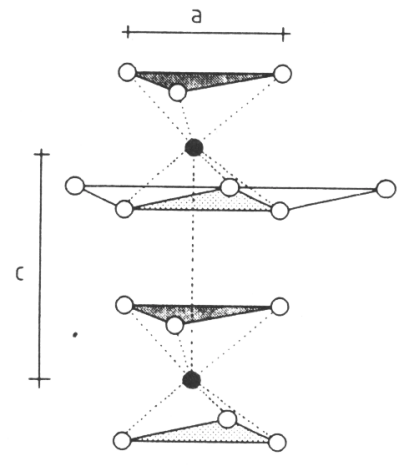

Figura 1. Estrutura do 1T-TiS 2 . Círculos preenchidos e vazios representam os átomos de titânio e enxofre, respectivamente. Os parâmetros de rede " $a$ " $e$ " $c$ " são indicados na figura.

Sulfetos e selenetos que possuem estruturas lamelares intercalam facilmente cátions orgânicos e inorgânicos em sítios vacantes nas lacunas de van der Waals, produzindo-se os compostos de intercalação do tipo $\mathrm{A}_{\mathrm{x}} \mathrm{MX}_{2}$ ou $\mathrm{A}_{\mathrm{x}}\left(\mathrm{H}_{2} \mathrm{O}\right)_{\mathrm{y}} \mathrm{MX} \mathrm{X}_{2}(\mathrm{M}=$ metal de transição, $\mathrm{X}=\mathrm{S}$ e $\mathrm{Se}, \mathrm{A}=$ cátion intercalado) ${ }^{1}$. Os principais processos de intercalação de cátions orgânicos derivados de aminas são: através do aquecimento da matriz lamelar com excesso de amina os quais são selados em ampolas de vidro $^{2,3}$, através de reações de troca iônica ${ }^{1}$ ou através da intercalação eletroquímica ${ }^{4}$. As vantagens do método eletroquímico são a detecção de intermediários de reação, síntese controlada de compostos com determinadas estequiometrias, além de geração de dados termodinâmicos e cinéticos, dados esses importantes para a compreensão dos mecanismos das reações envolvidas. Este método envolve a transferência de elétrons à matriz hospedeira, na qual os metais de transição envolvidos são submetidos a um processo de redução produzindose lamelas negativamente carregadas. Por balanceamento de cargas, cátions solvatados (ou não) da solução são alojados em sítios vacantes nas lacunas de van der Waals.

Neste trabalho serão apresentados resultados da intercalação eletroquímica de cátions hexilamínio hidratados, na fase $1 \mathrm{~T}-\mathrm{TiS}_{2}$, a partir de uma solução aquosa de sulfato de hexilamínio $0,5 \mathrm{Mol} / 1 \mathrm{em} \mathrm{pH}=5$. A utilização da técnica de voltametria cíclica permitiu observar dois compostos de intercalação. Estes compostos foram caracterizados por difratometria de raios-X, efetuando-se medidas dos espaçamentos interplanares basais. A partir destes estudos, representações esquemáticas do posicionamento dos cátions hexilamínio nas lacunas de van der Waals foram propostas.

\section{EXPERIMENTAL}

O dissulfeto de titânio na forma de um pó dourado foi preparado através da selagem dos materiais constituintes em ampolas de quartzo sob vácuo, aquecidas em forno tubular a uma temperatura de $640^{\circ} \mathrm{C}$ por 10 dias, utilizando-se iodo como agente transportante ${ }^{5,6}$.

A solução de sulfato de hexilamínio foi preparada titulando-se uma solução aquosa de hexilamina p.a. (Eastman Organic Chemicals) $0,5 \mathrm{Mol} / 1 \mathrm{com}$ ácido sulfúrico Merck até atingir um $\mathrm{pH}=5$. Os voltamogramas cíclicos foram obtidos utilizando-se cerca de $2 \mathrm{mg}$ de $1 \mathrm{~T}-\mathrm{TiS}_{2}$, o qual foi colado em uma chapa de platina de cerca de $1 \times 1 \mathrm{~cm}$ utilizando uma cola condutora de grafite (Leit-C - Neubauer Chemikalien). O eletrodo de trabalho assim constituído (após secagem por cerca de 15 minutos) foi mergulhado em uma cela de três compartimentos com cerca de $30 \mathrm{ml}$ de solução. Como contra eletrodo utilizou-se uma chapa de platina do mesmo tamanho do eletrodo de trabalho. Como referência utilizou-se um eletrodo de $\mathrm{Ag} / \mathrm{AgCl}(\mathrm{KCl}$ $1 \mathrm{Mol} / \mathrm{l})$. A temperatura de trabalho foi em torno de $20^{\circ} \mathrm{C}$.

As medidas ciclovoltamétricas foram realizadas utilizandose um potenciostato/galvanostato PARC 273A operando com um software comercial.

Os difratogramas de raios- $\mathrm{X}$ foram obtidos imediatamente após as amostras de $1 \mathrm{~T}-\mathrm{TiS}_{2}$ terem sido submetidas a uma varredura de potenciais e tempos de polarização de cerca de 2 horas. O difratômetro utilizado foi um Rigaku (20mA e 40kV) utilizando-se radiação $\mathrm{CuK} \alpha=1,5418 \AA$ e um monocromador de $\mathrm{LiF}$ situado entre a amostra e o detector. Os materiais foram alojados em um porta-amostras de vidro plano e submetidos a velocidades de varredura de $2 \%$ min. Utilizou-se em cada experimento um padrão interno de silício metálico. 


\section{RESULTADOS E DISCUSSÕES}

Na figura 2 estão representados quatro voltamogramas cíclicos consecutivos (1-4), referentes à intercalação dos cátions hexilamínio hidratados na matriz lamelar $1 \mathrm{~T}-\mathrm{TiS}_{2}$.

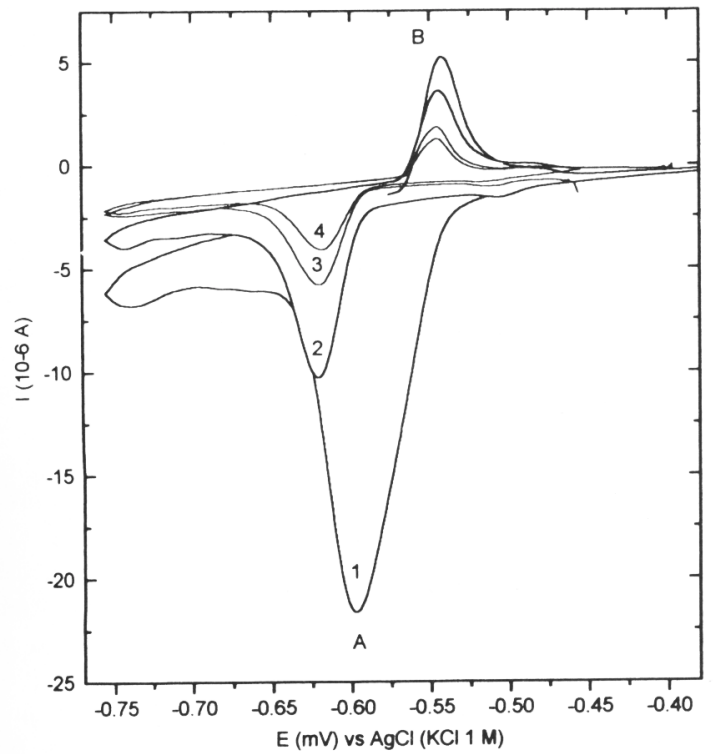

Figura 2. Voltamogramas cíclicos consecutivos da intercalação de cátions hexilamínio em $1 T$-TiS2 $(1-4=$ ciclos 1 à 4 , respectivamente). $A=$ primeiro pico de redução e $B=$ pico de oxidação. Velocidade de varredura igual a $0,15 \mathrm{mV} / \mathrm{s}$.

No primeiro ciclo é observado um pico largo de redução (A) com potencial de $0,597 \mathrm{~V}$ e um pico de oxidação (B) com potencial de $0,553 \mathrm{~V}$. A partir do segundo ciclo, observa-se um deslocamento do pico de redução para potenciais mais negativos. O mesmo efeito é observado com o pico de oxidação, porém em menor intensidade.

Em alguns experimentos foram observados ombros no pico de redução durante o primeiro ciclo, o que sugeriu a existência de vários processos ocorrendo em potenciais bastante próximos, dos quais somente alguns (ou 1) são reversíveis. Estes processos são sobrepostos apresentando-se na forma de uma banda larga.

Medidas cronopotenciométricas não foram possíveis pela pequena contaminação da fase $1 \mathrm{~T}-\mathrm{TiS}_{2}$ com outros sulfetos como uma fase rica em titânio $\left(\mathrm{Ti}_{1+\mathrm{x}} \mathrm{S}_{2}\right)$, entre outros. O nível de contaminação é bastante pequeno, sendo observados porém pequenos picos no difratograma de raios-X em $2 \theta=10,1$ e 26,7, não indexáveis na fase $1 \mathrm{~T}-\mathrm{TiS}_{2}$. Apesar da pequena contaminação, os parâmetros de rede do $1 \mathrm{~T}-\mathrm{TiS}_{2}$ são coincidentes com a literarura ${ }^{1}$. Outro fato observado é a liberação de gás sulfídrico durante a exposição do material por longos períodos ao ar. Apesar da exposição prolongada não foram observadas alterações nos difratogramas de raios-X.

As análises quantitativas não foram possíveis pela impossibilidade de quantificar os níveis de contaminação da fases rica em titânio e ocorrência da fase não intercalada mesmo após várias horas de polarização.

Os compostos de intercalação formados no processo de redução durante a voltametria cíclica foram isolados e caraterizados por difratometria de raios-X utilizando-se o método de pó (Fig. 3).

Estas medidas fundamentam-se basicamente na determinação da variação do espaçamento interplanar, isto é, o espaço ocupado pelo cátion alojado na lacuna de van der Waals, utilizando-se como referência o parâmetro de rede basal da matriz hospedeira vazia $\left(\Delta \mathrm{d}=\mathrm{d}_{\mathrm{Ax}(\mathrm{H} 2 \mathrm{O}) \mathrm{yTiS} 2}-\mathrm{c}_{1 \mathrm{~T}-\mathrm{TiS} 2}\right)$.
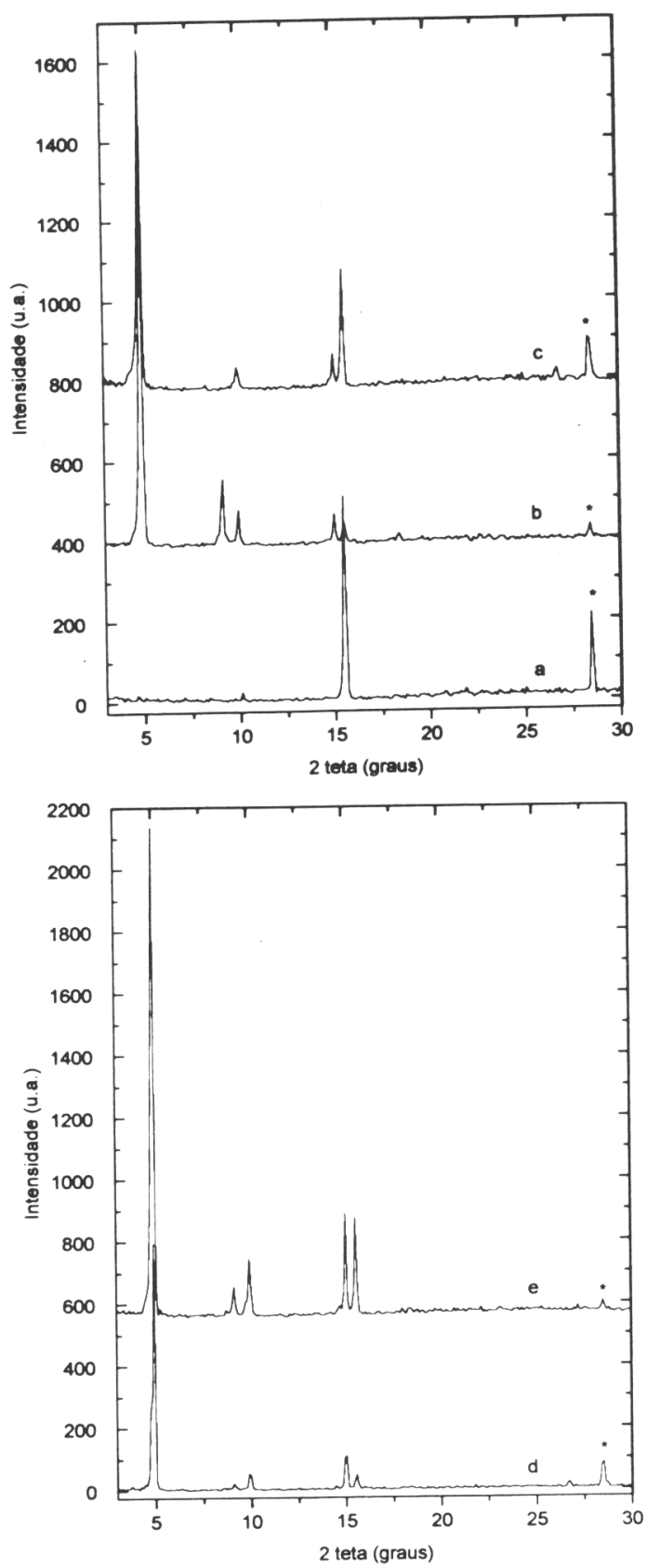

Figura 3. Difratogramas de raios- $X$ para amostras de $1 T-T i S_{2}$ intercaladas com cátion hexilamínio, em diferentes potenciais: (a) $1 T-T i S_{2},(b)$ $E=-0,56 \mathrm{~V}$, (c) $E=-0,62 \mathrm{~V}$, (d) $E=-0,70 \mathrm{~V}$, (e) $E=-0,45 \mathrm{~V}$. $O$ asterisco (*) mostra os picos relativos ao padrão interno de silício metálico.

Os materiais se mostraram bastante cristalinos na direção basal devido as interações eletrostáticas existentes entre as cadeias orgânicas positivamente carregadas e as lamelas, negativamente carregadas. Os materiais na forma de pequenas placas permitem uma excelente orientação preferencial dos cristalitos paralelamente ao porta-amostras de vidro. Todos estes fatores foram decisivos para que somente reflexões basais fossem observadas e os espaçamentos interplanares pudessem ser facilmente determinados.

Conhecendo-se o comprimento das cadeias orgânicas ou o espaço ocupado por esses cátions hidratados intercalados, importantes informações estruturais podem ser obtidas através de difratometria de raios-X, no que consiste à geometria molecular e posicionamento das cadeias das moléculas orgânicas nas lacunas de van der Waals. 
A tabela 1 apresenta os valores de $2 \theta$, espaçamentos interplanares basais (d) e as respectivas indexações (índices de Miller) e identificações das fases obtidas (difratogramas mostrados na figura 3 ).

Tabela 1. Valores de $2 \theta$, espaçamentos interplanares basais, indexações (índices de Miller) e identificações das fases obtidas (figura 3).

\begin{tabular}{|c|c|c|c|}
\hline Difratograma & $2 \theta$ (graus) & $\mathrm{d}(\AA)$ & Indexação \\
\hline \multirow[t]{7}{*}{$\mathrm{b}$} & 4,95 & 17,84 & 001 fase 1 \\
\hline & 9,16 & 9,65 & 001 fase 2 \\
\hline & 9,97 & 8,87 & 002 fase 1 \\
\hline & 15,00 & 5,91 & 003 fase 1 \\
\hline & 15,51 & 5,71 & $001-1 \mathrm{~T}-\mathrm{TiS}_{2}$ \\
\hline & 26,77 & 3,33 & $?$ \\
\hline & 28,49 & 3,13 & $111-\mathrm{Si}$ \\
\hline \multirow[t]{6}{*}{$\mathrm{c}$} & 4,90 & 18,04 & 001 fase 1 \\
\hline & 9,97 & 8,85 & 002 fase 1 \\
\hline & 15,04 & 5,89 & 003 fase 1 \\
\hline & 15,54 & 5,70 & $001-1 \mathrm{~T}-\mathrm{TiS}_{2}$ \\
\hline & 26,78 & 3,33 & $?$ \\
\hline & 28,49 & 3,13 & $111-\mathrm{Si}$ \\
\hline \multirow[t]{7}{*}{ d } & 4,90 & 18,03 & 001 fase 1 \\
\hline & 9,09 & 9,73 & 001 fase 2 \\
\hline & 9,94 & 8,90 & 002 fase 1 \\
\hline & 14,98 & 5,91 & 003 fase 1 \\
\hline & 15,52 & 5,71 & $001-1 \mathrm{~T}-\mathrm{TiS}_{2}$ \\
\hline & 26,67 & 3,34 & $?$ \\
\hline & 28,46 & 3,14 & $111-\mathrm{Si}$ \\
\hline \multirow[t]{6}{*}{$\mathrm{e}$} & 4,94 & 17,88 & 001 fase 1 \\
\hline & 9,15 & 9,67 & 001 fase 2 \\
\hline & 9,99 & 8,86 & 002 fase 1 \\
\hline & 15,01 & 5,90 & 003 fase 1 \\
\hline & 15,54 & 5,70 & $001-1 \mathrm{~T}-\mathrm{TiS}_{2}$ \\
\hline & 28,54 & 3,13 & $111-\mathrm{Si}$ \\
\hline
\end{tabular}

Fase $1-c=17,7 \AA$; Fase $2-c=9,7 \AA$.

Polarizando-se o $1 \mathrm{~T}-\mathrm{TiS}_{2}$ no potencial referente ao início do processo de redução (figura 2) observa-se uma reflexão grande no difratograma de raios- $X$ correspondente a um composto com parâmetro de rede basal $c=17,7 \AA$ (fase 1 ), uma reflexão pequena com $c=9,7 \AA$ (fase 2 ), além de reflexões correspondentes ao retículo hospedeiro vazio $1 \mathrm{~T}-\mathrm{TiS}_{2}$ e silício metálico (Fig. 3(b)).

No meio e no final do processo de redução (Fig. 2), o difratograma de raios-X praticamente se repete mostrando que a fase predominante é aquela que apresenta um parâmetro de

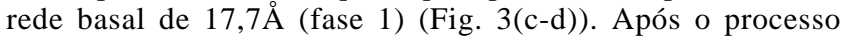
de oxidação (Fig. 2), o difratograma apresenta um aumento relativo das reflexões correspondentes à fase com parâmetro de rede basal de 9,7 (fase 2) (Fig. 3(e)). Em praticamente todos os difratogramas observou-se uma reflexão relativa a fase original provavelmente não reagida pelo fato de não estar ligada eletricamente ao eletrodo de trabalho, submersa na cola condutora o que impediu a reação ou desconectada após o início da reação (através do inchamento do cristal).

Quando a amostra é submetida à dois ciclos completos e retirada no potencial relativo à oxidação observa-se um difratograma de raios- $\mathrm{X}$ bastante semelhante aquele observado após o primeiro ciclo completo. Uma explicação para os dois compostos observados através da ciclovoltametria é fundamentada basicamente nas observações realizadas através de difratometria de raios-X.

O comprimento da cadeia do cátion hexilamínio isolado e não hidratado calculado através do programa de modelagem molecular Alchemy ${ }^{7}$ é de $8,8 \AA$. O programa foi utilizado para construir uma molécula livre, utilizando as distâncias interatômicas e ângulos de ligação médios do próprio programa, obtidos na literatura. Dividindo este valor pelo número de átomos de carbono teremos $1,467 \AA ̊$ Átomo de carbono na cadeia. Este valor é coerente com os dados obtidos da intercalação térmica de $1 \mathrm{~T}-\mathrm{TiS}_{2}$ e $2 \mathrm{H}-\mathrm{NbS}_{2}$ com octadecilamina e octilamina intercaladas em dupla camada, os quais apresentam espansões de 52,66 e $23,47 \AA$, respectivamente ${ }^{2}$. Estes valores correspondem a $1,463 \AA$ átomo de carbono na cadeia no caso da octadecilamina e $1,467 \AA$ /átomo de carbono na cadeia no caso da octilamina, portanto coerentes com o valor obtido através do cálculo acima.

Representações esquemáticas referentes ao posicionamento dos cátions hexilamínio na rede hospedeira de $1 \mathrm{~T}-\mathrm{TiS}_{2}$ foram propostos neste trabalho (Fig. 4), baseados nos resultados descritos acima e considerando que: i) o parâmetro de rede basal do $1 \mathrm{~T}-\mathrm{TiS}_{2}$ é de $5,7 \AA^{1}$, ii) o diâmetro de van der Waals da molécula de água é de $3 \AA^{1}$, iii) o diâmetro da cadeia carbônica é de aproximadamente $4 \AA$, e, iv) o cátion hexilamínio foi intercalado com uma camada de moléculas de água de hidratação, como ocorre com cátions de metais alcalinos e amônio intercalados em $1 \mathrm{~T}-\mathrm{MoS}_{2}{ }^{8-10}, \quad 2 \mathrm{H}-\mathrm{NbS}_{2}{ }^{1}$ e $2 \mathrm{H}-\mathrm{TaS}_{2}{ }^{1}$.

Para os cálculos das variações das expansões interplanares fez-se a substração entre o parâmetro de rede basal observado e o parâmetro de rede basal da matriz original não intercalada ou seja $5,7 \AA^{1}$.

Observe-se que na figura 4 estão representados os posicionamentos esquemáticos dos cátions hidratados intercalados na lacuna de van der Waals sem levar em consideração as proporções dos tamanhos dos átomos envolvidos.

$\mathrm{O}$ cátion hexilamínio hidratado posicionado paralelamente às lamelas ocasionaria uma expansão interplanar de $4 \AA$. Como no início do processo de redução foram observados compostos com expansões interplanares de $4 \AA(9,7 \AA$ da fase obtida menos $5,7 \AA$ da matriz hospedeira vazia) e $12 \AA(17,7-5,7)$, admitiu-se que nestes casos os cátions hidratados são intercalados nas lacunas de van der Waals, paralelamente às lamelas (Fig. 4(1)) e perpendicularmente (Fig. 4(2)), respectivamente.

Na mistura predomina o composto com parâmetro de rede basal de $17,7 \AA$. O comprimento do cátion hexilamínio hidratado intercalado perpendicularmente às lamelas nas lacunas de van der Waals e direcionados alternadamente para baixo e para cima (ou não), como demonstrado na figura 4-2, ocasionaria uma expansão interplanar de $11,8 \AA ̊$. Após o processo de oxidação regenera-se parcialmente a fase correspondente a $4 \AA$, pelo retomada do posicionamento das moléculas intercaladas paralelamente as lamelas, embora este processo seja somente parcialmente reversível (Fig. 2 e 3(e)). Este fato se deve principalmente pela diminuição da concentração dos cátions nas lacunas de van der Waals.

Observa-se em alguns difratogramas de raios-X (Fig. 3(c)) uma assimetria no primeiro pico, provavelmente mostrando a presença de uma fase com parâmetro de rede basal maior que $17,7 \AA$, possívelmente devido a formação de estágios. 
(1)

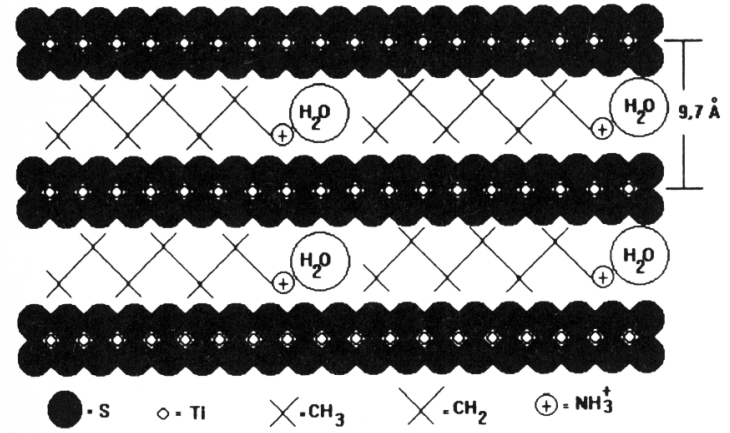

(2)

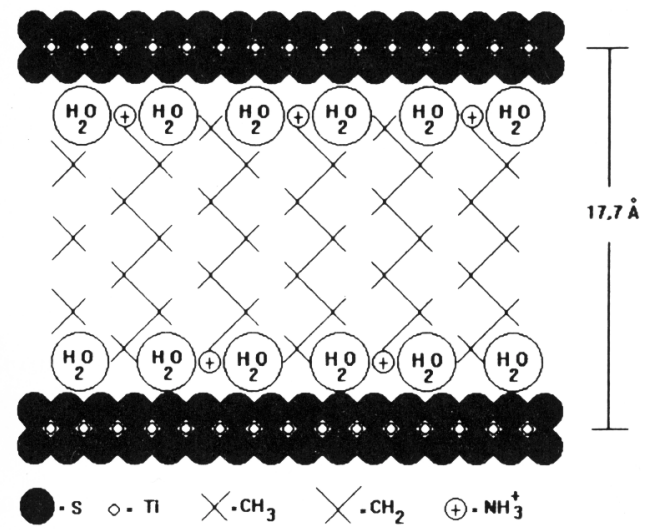

Figura 4. Representações esquemáticas do posicionamento dos cátions hexilamínio hidratados intercalados nas lacunas de van der Waals do composto $1 T-T i S_{2}$, paralelamente (1) e perpendicularmente às lamelas (2), respectivamente.

\section{CONCLUSÕES}

Os valores das expansões interplanares calculados são bastante próximos dos valores observados por difratometria de raios-X. As representações esquemáticas dos cátions hidratados posicionados paralelamente às lamelas (calculado $4 \AA$ e observado $4 \AA$ ), dos cátions hidratados e posicionados perpendicularmente às lamelas na forma de monocamada (calculado $11,8 \AA$ e observado $12 \AA$ ) podem ser interpretadas como:

No pico de redução largo os cátions hexilamínio hidratados intercalados são posicionados paralelamente. A partir de um pequeno aumento de potencial de redução, os cátions passam a se posicionar perpendicularmente às lamelas na forma de monocamada, principalmente pelo aumento da concentração dos cátions.
- No pico de oxidação, onde observa-se uma pequena reversibilidade, esta é associada principalmente à reação que leva o composto à fase com as moléculas posicionadas paralelamente às lamelas. Isto devido provavelmente ao processo de "tombamento" das moléculas intercaladas provocado pela diminuição da concentração das mesmas.

A existência das fases hospedeira inicial $\left(1 \mathrm{~T}-\mathrm{TiS}_{2}\right)$ e as fases com cátions posicionados paralelamente e perpendicularmente as lamelas, nos dois picos de corrente podem ser explicadas por uma série de fatores ou seja: problemas difusionais onde pequenas ilhas podem ser produzidas no interior do cristal; tempo insuficiente no processo de polarização; possível pobre contacto elétrico com o eletrodo de trabalho durante o processo de fixação da matriz; a possibilidade da submersão da matriz na cola condutora o que impediu a reação ou ainda o descolamento dos cristais após os primeiros passos de reação (inchamento do cristal).

Há ainda a possibilidade de que o pico de redução esteja ligado a uma transição estrutural irreversível, que poderia estar alterando o empilhamento das lamelas da matriz hospedeira original. Esta hipótese é respaldada pela menor energia envolvida em transladar ou rotar as placas, devido à grande expansão interplanar provocada pelos cátions hexilamínio hidratados. Resultados semelhantes foram observados na intercalação de cátions hexilamínio na matriz lamelar $2 \mathrm{H}-\mathrm{NbS}_{2}{ }^{11}$.

\section{REFERÊNCIAS}

1. Subba Rao, G. V.; Shafer, M. W.; Intercalated Layered Materials, vol. 6 (Ed. Levy, F.), D. Reidel Publishing., Dordrecht, 1979.

2. Schöllhorn, R.; Sick, E.; Weiss, A.; Z. Naturf. 1973, 28b, 168.

3. Weiss, A,; Ruthardt, R., Z. Naturf. 1973, 28b, 249.

4. Tagaya, H.; Ara, K.; Ogata, T.; Matsushita, K.; Kadokawa, J.; Karasu, M. and Chiba, K.; Chem. Letters 1994, 2439.

5. Gentilmente cedido pelo Dr. Karsten Sollmann do Departamento de Química da Universidade Técnica de Berlim Alemanha.

6. McKelvy, M. J.; Glausinger, W. S.; Mat. Res. Bul. 1986, 21,835 .

7. Alchemy II - Tripos Associates, Inc., 1988.

8. Wypych, F.; Tese de Doutorado, 1992, PUC/RJ.

9. Wypych, F.; Tsunoda, M.; Quím. Nova 1994, 17, 9.

10. Wypych, F.; Sollmann, K.; Schöllhorn, R.; Mat. Res. Bull. 1992, 27, 545.

11. Wypych, F.; Biason Gomes, M. A.; Denicoló, I.; Barreto Adad, L.; J. Electrochem. Soc., no prelo. 